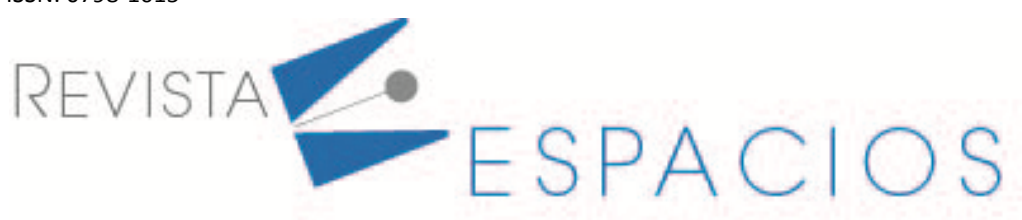

\title{
Modelo conceptual para la creación de valor en el turismo rural comunitario
}

\section{Conceptual model for the creation of value in rural community tourism}

\author{
CASTAÑEDA TORRES, Solanyie ${ }^{1}$ \\ USSA GARZÓN, Jaime Eddy ${ }^{2}$ \\ RODRÍGUEZ MIRANDA, Juan Pablo ${ }^{3}$
}

\begin{abstract}
Resumen
Surgen nuevas alternativas de turismo, siendo el turismo rural comunitario fundamental como herramienta de desarrollo económico en países de bajos ingresos y de altos activos naturales. Teniendo en cuenta el conocimiento, experiencia de guías y agencias de turismo se obtuvieron aspectos de cadena de valor, el objetivo propone un modelo conceptual para la cadena de valor de turismo rural comunitario que articule aspectos tangibles e intangibles en la oferta total de la cadena valor. En resumen, el turismo rural comunitario no ha sido explorado en su totalidad, se recomienda articular con actores locales en la creación de valor.

Palabras clave: turismo rural, comunitario, creación de valor, patrimonio, accesibilidad, modelo.
\end{abstract}

\begin{abstract}
New alternatives for tourism arise, being the fundamental community rural tourism as a tool of economic development in low income countries and high natural assets. Taking into account the knowledge, experience of guides and tourism agencies, aspects of the value chain were obtained, proposing a conceptual model for the rural community tourism value chain that articulates tangible and intangible aspects in the total supply of the value chain. In summary, rural community tourism has not been explored in its entirety, it is recommended to coordinate with local actors in the creation of value. Key words: rural tourism, community, value creation, heritage, accessibility, mode
\end{abstract}

\section{Introducción}

Como es natural, el turismo enfrenta fuerte demanda y compromiso débil por parte de comunidades locales (Unwto \& Snv, 2010). Las acciones negativas aumentan la vulnerabilidad de otros sistemas (Barnett \& O'Neill, 2010). En varios escenarios, influyen factores por falta de capital financiero, escaza organización, exclusión de intereses en el sector formal, poco poder de regulación y negociación, baja oferta de servicios turísticos y falta de apoyo del sector gubernamental entre otros factores, recibiendo críticas en patrones de comportamiento y

\footnotetext{
${ }^{1}$ M. Sc. Desarrollo Sustentable y Gestión Ambiental. Investigadora Grupo de investigación - Aquaformat. Universidad Distrital Francisco José de caldas. Bogotá, Colombia. Dirección: Correo electrónico: sct.sustentable@gmail.comAvenida Circunvalar Venado de Oro. Bogotá D.C. Colombia. ${ }^{2}$ Profesor Asistente. Facultad del Medio Ambiente y Recursos Naturales. Universidad Distrital Francisco José de Caldas. Correo electrónico: jeussag@udistrital.edu.co ${ }^{3}$ Profesor Titular. Facultad del Medio Ambiente y Recursos Naturales. Universidad Distrital Francisco José de Caldas. Correo electrónico: jprodriguezm@udistrital.edu.co
} 
las estructuras culturales (Kyungmi \& Muzaffer, 2013; Unwto \& Snv, 2010). Es necesaria la planificación y gestión del turismo prestando atención a componentes locales como el paisaje (Carneiro, M. J., Lima, J., \& Silva, A. L. 2015).

En este sentido, surgen nuevas alternativas de turismo comunitario, como lo menciona Goodwin (2009), el turismo rural y ecoturismo, son alternativas fundamentales como herramientas de desarrollo y reducción de pobreza. Es importante, crear valor específicamente en el factor de calidad en servicios es una tarea ardua y los segmentos de mercado son altamente competitivos (Scaglione, Marx y Johnson, 2011). El turismo se establece como herramienta desarrollo económico en la región, a su vez, generador de oportunidades de empleo local y bienestar social. Esté se centra en el desarrollo hacia países con bajos ingresos, activos naturales, es decir, requiere poco capital de inversión y relativamente pocas habilidades para su desarrollo (Unwto \& Snv, 2010). Si bien es cierto, el turismo es generador de empleo mediante el fomentó en creación de empresas que aseguran fuentes de ingreso a grupos menos favorecidos (Unwto \& Snv, 2010). En realidad, los beneficiarios del turismo social son personas que presentan características de falta de dinero, problemas de salud y/o condiciones de discapacidad (Goodwin, 2009).

El turismo rural se define por la Organización Mundial del Turismo UNWTO (por sus siglas en inglés) como: el turismo rural es un tipo de actividad turística en el que la experiencia del visitante está relacionada con un amplio espectro de productos vinculados por lo general con las actividades de naturaleza, la agricultura, las formas de vida y las culturas rurales, la pesca con caña y la visita a lugares de interés (OMT, 2019). Cabe señalar, las empresas pequeñas de turismo tienen un rol importante en el desarrollo rural de la comunidad, contribuyen a la multiplicación de ingresos. La industria del turismo es accesible a familias y/o personas que buscan diversificar las fuentes de ingresos, además, brinda oportunidades en torno a los destinos rurales y mejora la calidad de vida de los habitantes (Po-Hsin, Morrison-Saunders, \& Grimstad, 2017).

Si bien, el éxito en la perspectiva de creación de valor depende de estrategias diferenciales que examinen el valor qué los clientes están buscando, la satisfacción es medida en actitudes, comportamientos y capacidad de los empleados. Hasta ahora, los beneficios empresariales ofrecidos a los clientes afectan la decisión de quedarse o cambiar de proveedor, es decir, basan su percepción en la hipótesis de las experiencias del servicio (Kyungmi \& Muzaffer, 2013). La cadena de beneficios en la prestación de servicios muestra la relación entre la capacidad del empleado, beneficios empresariales y satisfacción al cliente (Kyungmi \& Muzaffer, 2013). El encuentro durante el servicio es esencial en la experiencia de los usuarios, esté juega un papel importante, creando innovación y conocimiento (Sørensen, Mattsson, \& Sundbo, 2013). No obstante, la adquisición de información de sus usuarios activa atributos que facilitan experiencias turísticas y enfatizan en aspectos de calidad en el servicio y reducción de costos (Sørensen \& Jensen, 2015). El objetivo del presente artículo propone el desarrollo de un modelo conceptual para la creación de valor en la cadena de turismo rural comunitario.

\subsection{Patrimonio como impulsor de turismo}

En efecto, el impacto del turismo se puede analizar desde varios enfoques: ambiental, económico, social y cultural. El turismo es entendido por el fenómeno de activos patrimoniales existentes, la investigación identifica el patrimonio como impulsor de turismo y como principal factor de desarrollo; definido como el proceso social en que las partes locales que se encuentran interesadas construyen identidad nacional a partir de tomadores de decisión, autoridades, y actores sociales (Zahra, Gedajlovic, Neubaumc, \& Shulmand, 2009). En proceso de la globalización, cambia el contexto, en la contribución de cambiar la naturaleza, y se globaliza el patrimonio (Gravari-Barbas, 2018).

Por lo tanto, la política económica, las tradiciones judiciales y la crisis económica impulsan al desarrollo de emprendimiento social (Altinay, Sigala, \& Waligo, 2016). El emprendimiento social es un fenómeno basado en 
procesos a través de recursos intangibles, capital social y humano, que busca oportunidades para identificación de valor (Lin, Chen, \& Filieric, 2017). Comprende actividades para definir, descubrir y explotar oportunidades mejorando la riqueza social en la creación de nuevas empresas y organizaciones que apunten a la innovación (Zahra, Gedajlovic, Neubaumc, \& Shulmand, 2009). Los actores interactúan en intercambio de recursos en la cocreación de valor para ellos y otros, aportando a la mejora y bienestar comunitario (Vargo \& Lusch, 2011). De hecho, el turismo social conduce al cambio social siendo un motor importante para la creación de valor (Kline, Shah, \& Rubright, 2014). En consecuencia, el nuevo enfoque adopta la generación de valor social y el servicio sostenible (Enquist, Sebhatu, \& Johnson, 2015). En la perspectiva dominante de servicio está el conocimiento y la participación de actividades empresariales en el turismo social (Vargo \& Lusch, 2011). De manera que, el turismo se trata de personas que interactúan fuera de casa con otros entornos y lugares(Sharpley, 2014).

Este tipo de experiencias e interacciones influencian en el bienestar, la satisfacción y comportamientos futuros (Sharpley, 2014). El tejido comunitario se representa en la forma de participación dinámica en el desarrollo de sentido de pertinencia. Obteniendo compromisos y responsabilidades en la articulación de amistad y afinidad (Flórez Ñustes \& Laguna Barragán, 2017). De lo anterior, estudios relacionados dirigen las investigaciones hacia la satisfacción con la vida o bienestar de los turistas (Dolnicar, Yanamandram, \& Cliff, 2012), según Liang \& Hui (2016), indagan acerca del apoyo para el desarrollo turísticos y la calidad de vida. Es oportuno ahora, definir el valor en términos de costo - beneficio considerado como compensación y esté resultado procede de los atributos del servicio. En efecto, la coproducción implica el diseño de servicios y productos a los turistas, donde el proveedor del servicio de turismo se percibe como productor de resultados valiosos para los turistas (Rihova, Buhalis, Moital, \& Gouthro, 2015). De modo que, los turistas juegan un papel activo en la búsqueda y determinación de experiencias memorables ( Ritchie \& Hudson, 2009).

El valor del turismo se define de las experiencias con la interacción, intercambio y valor recíproco creado ( Ritchie \& Hudson, 2009). Seguido, al valor de uso donde los turistas determinan y evalúan el producto y servicio según sus experiencias. Por esta razón, existen dimensiones de cooperación en creación de valor: coproducción y valor de uso (Rihova, Buhalis, Moital, \& Gouthro, 2015). El impacto del turismo se puede analizar desde varios enfoques: ambiental, económico, social y cultural; dentro de los territorios están construidos elementos estos relacionan prácticas que institucionalizan y legitimarán el sentido de apropiación (Ocampo González, 2017). El turismo es entendido por el fenómeno de activos patrimoniales existentes, la investigación identifica el patrimonio como impulsor de turismo y como principal factor de desarrollo; definido como el proceso social en que las partes locales que se encuentran interesadas construyen identidad nacional a partir de tomadores de decisión, autoridades, y actores sociales (Zahra, Gedajlovic, Neubaumc, \& Shulmand, 2009). En proceso de la globalización, cambia el contexto, en la contribución de cambiar la naturaleza, y se globaliza el patrimonio (Gravari-Barbas, 2018).

Si bien es cierto, las comunidades rurales están excluidas en el desarrollo urbano, representando limitantes en el acceso de oportunidades, recursos humanos y capacidades. Sin embargo, el potencial de turismo está dado en el subdesarrollo de las zonas rurales que ofrecen tranquilidad, paz y belleza. En cierto sentido, proporciona empleo en espacios rurales y se asocia a mano de obra no calificada (Ashish, Umesh, \& P. Vigneswara, 2017).

\subsection{La accesibilidad del turismo social}

En el siguiente lugar, se encuentra el turismo en masas, y la visión de nuevas prácticas turistas ( Fiorello \& Bo, 2012 ; Jepson \& Sharpley, 2015 ). El turismo rural se ha transformado en un negocio complejo simultáneamente es un atractivo múltiple en el mercado (Lane \& Kastenholz, 2015). Por ello, el papel de "Red de relaciones" adquiere importancia central, en los sectores públicos y privados, así como los residentes locales (Corigliano et al., 2015). Las oportunidades de desarrollo, favorece los recursos locales de la economía tradicional, enfrentando el desafío de convertirse en destinos cada vez más atractivos (Chaperon \& Bramwell, 2013 ). Ahora bien, no sólo 
se incluye el turismo rural sino el ciclismo de montaña, actividades de senderismo, turismo gastronómico, turismo de bienestar y activo, los turistas van atraídos por la búsqueda de un valor experiencial (Salvatore, 2015).

El turismo social como valor agregado beneficia directamente al visitante en el intercambio de experiencias, integrando necesidades y al mismo tiempo, facilitando la participación del turismo en la mejora de la percepción social (Sedgley, Pritchard \& Morgan, 2012 ). En primera instancia, el mercado debe garantizar competitividad a largo plazo y generar valor agregado ( Pulido- Fern Ández et al., 2014 ). Derivando beneficios en el fomento de empresas rurales, mejora de infraestructura, y preservación e intercambio cultural (Wang \& Chen, 2015). A través de la participación de lugareños y los efectos para cumplir los propósitos, involucra a la comunidad a desarrollar consciencia de los beneficios puede aportar el turismo (Rasoolimanesh, Jaafar, Kock, y Ramayah, 2015).

Los procesos de empoderamiento y planificación en el turismo por parte de los residentes involucran la participación de la comunidad, siendo elemento principal para iniciativas claves (Stylidis, Biran, Sit, y Szivas, 2014; Woo et al., 2015). Las comunidades son los custodios para preservar, conservar y defender los recursos culturales no renovables (Mustafa \& Tayeh, 2011). El apoyo por parte de los residentes garantiza el desarrollo sostenible del turismo local y determina la calidad de los visitantes (Woo, Kim, \& Uysal, 2015 ). Es necesaria, la participación de la comunidad a través de la implementación de propuestas adaptadas a necesidades locales que brinden oportunidades (Tolkach \& King, 2015). No obstante, existen dimensiones en el turismo que respaldan a los residentes y están enfocadas hacia el mercado, la sociedad y el desempeño económico (Camarero, Garrido, \& Vicente, 2015). En términos sociales el turismo es un factor de inclusión social, según Sedgley et al. (2012), los productos turísticos deben asociarse con diferentes niveles a turistas experimentados y turistas inexpertos.

De lo anterior, existen las categorías de turistas internacionales sin previa experiencia y turistas nacionales inexpertos, la inexperiencia y la falta de conocimiento vincula a los niveles de incertidumbre a la hora de tomar decisiones, en lugar de certeza, se usa el término de riesgo. Esto hace referencia entre lo conocido y desconocido, estos niveles de riesgos están determinados por género, edad, cultura y nivel socioeconómico, estimando preferencia por lo conocido y atadura a la ambigüedad ( Williams \& Baláz , 2012 ).

Si bien es cierto, el turismo es considerado como estimulador de la economía, durante el año 2012 registro el $5 \%$ del PIB mundial, representando importancia en factores de producción, mejora de las rutas de transporte, y estimulo de la economía en países emergentes. En materia de empleo, contribuye a sectores en mano de obra y mitiga el desempleo favoreciendo con posibilidades a mujeres, jóvenes (Ursache, 2015). Existen barreras que afectan las capacidades locales en la planificación de medio y largo plazo (Klint et al., 2012). En realidad, las empresas turísticas ofertan actividades de acceso integral y se centran en personas con discapacidades (Clemente Soler et al., 2018). Se destaca el turismo social por la escasez de recursos económicos con la intervención de agentes operadores que maximizan el beneficio colectivo. De esta manera, actividades estrategias de marketing se genera en la red colectiva de emprendedores, comerciantes y artistas (García Henche \& Salvaj Carrera, 2017). Estos factores de desarrollo hacia nuevos horizontes de accesibilidad garantizan bienestar, calidad humana, estableciéndose como un factor de ordenamiento territorial, económico, social, y de desarrollo mundial (Clemente Soler et al., 2018).

Ahora bien, la calidad de territorio configura el factor esencial para la competitividad de los destinos de turismo en el escenario mundial, enmarcado por estrategias de diferenciación, mediante representación paisajística diferenciada (Santos-Pavón, Fernández-Tabales, \& Muñoz-Yules, 2016). Aunque, el turismo natural y rural, depende directamente de los recursos naturales, constituyen un factor esencial y prioritario (Scott et al., 2012; Gómez Martín et al., 2017). En la creciente preocupación a hacer frente al cambio climático con estrategias de mitigación y adaptación como el turismo de bajo impacto (Becken et al., 2014). 
Por otro lado, el turismo social accesible es un nuevo modelo de turismo que promueve iniciativas de participación activa de personas con necesidades especiales (Garcés \& Ferri, 2012). Actualmente, los turistas buscan experiencias únicas como una prolongación de vida, mostrando interés por turismo de contacto natural que genere tranquilidad y estás experiencias son el verdadero producto (Cuenca \& Prat, 2012). En la articulación de turismo emocional y experiencial como integrador de tradiciones, cultura convirtiéndose en materia prima esencial (García Henche \& Salvaj Carrera, 2017). En efecto, el turismo es un bien social debe estar al alcance de todos los ciudadanos, el concepto de accesibilidad turística se refiere al servicio o instalación facilite el uso y disfrute de cualquier persona, independiente de su capacidad sensorial, cognitiva y física. Se refiere a la facilidad en el uso y disfrute de instalaciones físicas de manera autónoma, sin apoyarse en medidas especiales; está accesibilidad es necesaria para personas mayores, niños, personas con enfermedades, y con funcionalidad diferente (Clemente Soler et al., 2018).

En este orden, el turismo es considerado como exportación o importación, se relaciona con bienes y servicios que los turistas acceden durante el movimiento de un país, se denomina exportación. El gasto realizado por el turista en el extranjero para su país de residencia, se considera como importación. De modo que, el incremento de los turistas internacionales incrementa el comercio internacional, a su vez, estimula y fortalece la competitividad de empresas locales, direccionando mayor inversión (Ursache, 2015). No obstante, las remesas son fuentes de ingresos para los países en desarrollo, y potencializan otros sectores de la economía. En consecuencia, según Mincui (2004) el impacto del gasto turístico se mide en tres elementos: directo, indirecto e inducidos. El efecto directo se relaciona con el gasto en hoteles, restaurantes, comunicaciones, siendo generador de empleo. El indirecto en consumo de bienes y servicios adquiridos en la cadena de suministro de turismo. Por último, la inducida mide el PIB y el gasto directo e indirecto del turismo (Ursache, 2015).

\subsection{Segregación social en la cadena de valor de turismo rural comunitario}

La planificación y el apoyo a los residentes, se relaciona con beneficios percibidos y necesidades satisfechas mejorando la calidad de vida (Jaafar, Noor, \& Rasoolimanesh, 2015; Nunkoo \& So, 2015). El enlace de participación local y beneficios percibidos fomenta equidad, responsabilidades que se adaptan a oportunidades locales (Tolkach \& King, 2015). Está disposición de los residentes están influenciados por el bienestar social, mercado y económico (Liu et al., 2014). El proceso de turismo debe fortalecer el empoderamiento de la comunidad hacia participación en toma de decisiones enmarcadas a prosperar en el largo plazo, la participación de la comunidad en la política hace referencia a los lugareños (Prabhakarana, Naira, \& Ramachandranb, 2014 ; Boley \& McGehee, 2014). Está relación implica creación de empresas, mejora de condiciones laborales, construcción de infraestructura y contribución al desarrollo en la economía local, el desarrollo de actividades culturales e intercambio con visitantes equipara consciencia en los beneficios que atrae el turismo (Jaafar et al., 2015).

La ventaja de adaptación que desarrollan las comunidades para enfrentar los cambios potencialmente drásticos en una economía que fluctúa, la mayor parte de las ganancias están determinadas en una temporada del año (Butler et al., 2014a). Por lo tanto, las comunidades quienes preservan, conservan, salvaguardan y defienden los recursos culturales no renovables ( Mustafa \& Tayeh, 2011). Los sentimientos de valor, conexión, identidad de lugar, dependencia de lugar, preservan la cultura y son características atractivas para los visitantes internacionales (Blasco López, Recuero Virto, Aldas Manzano, \& García-Madariaga Miranda, 2018).

\subsection{Los objetivos de Desarrollo Sostenible como sustento del turismo rural comunitario}

El histórico acuerdo sobre la universal Agenda 2030 de Desarrollo Sostenible, alcanzado en 2015 por los líderes mundiales en las Naciones Unidas, supone que 196 países se comprometen a avanzar hacia la consecución de una serie de 17 Objetivos de Desarrollo Sostenible (ODS), en aras de un futuro mejor para todos (OMT, 2018). 
En consecuencia, la implementación de estrategias para obtener mejores resultados ambientales y humanos, se crea la capacidad de adaptación en las comunidades (Butler et al., 2014b). Al tomar un enfoque de sistemas, en la construcción reciente de vías de adaptación e inclusión en principios, valores, y objetivos; diseña e implementa estrategias transformadoras, coordinadas a escalas espaciales, sectoriales, y jurídicas (Rodima-Taylor et al., 2012). El uso eficiente de la tecnología origina mejores experiencias, siendo atractivo a turistas que desconocen lugares para el desarrollo de turismo. Esto indica la importancia de las redes sociales en las empresas turísticas, está combinación es relativamente nueva en la forma de comunicación y de interacción (Ashish, Umesh, \& P. Vigneswara, 2017). Los motores de búsqueda permiten el acceso con sitios web populares, impulsando ventajas competitivas en la toma de decisiones, esto crea experiencias colectivas para otros (Fotis, Buhails, \& Rossides, 2012). En la actualidad las redes sociales, ofrecen aplicaciones basadas en la innovación permitiendo a los usuarios la generación de contenido (Kaplan, 2010).

El número de usuarios facilita la interacción del turismo en línea, cumpliendo el papel de articulación entre turistas y agencias de viaje (Rodima-Taylor et al., 2012). En efecto, los viajeros globales reducen la incertidumbre en relación a los planes de viaje, mejorando las experiencias y beneficios del viaje (Kaplan, 2010). Es decir, los usuarios son quienes promueven mediante la generación de contenido antes y después de la experiencia, las redes sociales aportan en el imaginario de lugares para visitar de manera particular (Ashish, Umesh, \& P. Vigneswara, 2017). En consecuencia, comparten sus experiencias personales en el intercambio de comentarios relacionados a servicios adquiridos con el turismo (Mariani, Mura, \& Di Felice, 2017). De esta manera el turismo rural contribuye a los ODS 10 reducir la desigualdad en y entre los países, enfocándose en alternativas para la ruralidad que se sustentan en una cadena de valor diversificada al contar con un abanico amplio de destinos turísticos, con múltiples atractivos (productos turísticos), que satisfacen la necesidad de relacionarse con la naturaleza de las sociedades modernas, la calidad de los sitios se convierten en un reto de conservación y manejo, para que el marketing de destino se mantenga, la capacidad de carga no se supere y se logre la competitividad y calidad que requiere el destino turístico, esto solo es posible con la innovación permanente en este sector y en los productos que se convierten en centro de visita.

Sin embargo, el turismo aparece en las metas de los Objetivos 8, 12 y 14, sobre desarrollo económico sostenible e inclusivo, producción y consumo sostenibles, y uso sostenible de los océanos y de los recursos marinos, respectivamente. Ahora bien, gracias a su alcance y a su impacto, el turismo puede ayudar de forma directa o indirecta a lograr cualquiera de estos 17 Objetivos (OMT, 2018).

\section{Metodología}

La metodología aplicada en este trabajo, consistió en el desarrollo de un cuestionario semiestructurado para guías y agencias de turismo, en la selección del método mixto en tipo de investigación descriptiva y explicativa. Luego, se hizo el cálculo por muestreo aleatorio simple con guías y agencias de turismo, expresado en la tabla 2: 
Tabla 1

Selección de la muestra

\begin{tabular}{|l|l|}
\hline \multicolumn{1}{|c|}{ Muestreo aleatorio simple } & Donde: \\
\hline$n=\frac{z^{2} \cdot \mathrm{N} \cdot p \cdot q}{E^{2}(\mathrm{~N}-1)+z_{\alpha}^{2} \cdot p \cdot q}$ & $\begin{array}{l}\text { n: es el tamaño de la muestra; } \\
\text { Z es el nivel de confianza del } 95 \% . \\
\text { p es la variabilidad positiva; } \\
\text { q es la variabilidad negativa; } \\
n=\frac{(1.96)^{2} \cdot 28 \cdot 0,5 \cdot 0,5}{(0.07)^{2}(27-1)+(1.96)^{2} \cdot 0,5 \cdot 0,5}\end{array}$ \\
$\mathrm{E}$ es la precisión o error \\
\end{tabular}

Posteriormente, se consultaron guías y agencias de turismo; conformados por organizaciones que ofertan productos, servicios para el consumo y disfrute de destinos turísticos. Por consiguiente, se establece la representación acerca de la muestra seleccionada en la siguiente tabla 2 :

Tabla 2

Público objetivo

\begin{tabular}{|c|c|c|}
\hline Sector de turismo & \multicolumn{2}{|c|}{ Población } \\
\hline Enfoque & Número & Porcentaje \\
\hline Agencias de turismo & 15 & 60 \\
\hline Guías de turismo & 9 & 40 \\
\hline Total & 24 & $100 \%$ \\
\hline
\end{tabular}

Para el desarrollo del cuestionario semiestructurado, se identifican variables independientes y dependientes en relación con el turismo rural comunitario con el propósito crear la cadena de valor mediante un modelo conceptual. Desglosando las variables en la tabla 3:

Tabla 3

Variables de análisis de turismo rural comunitario.

\begin{tabular}{|c|c|c|c|}
\hline \multicolumn{2}{|c|}{ Variable independiente } & \multicolumn{2}{|c|}{ Variable dependiente } \\
\hline Aspectos tangibles & Aspectos intangibles & Aspectos tangibles & Aspectos intangibles \\
\hline VIAT & VIAI & VDAT & VDAI \\
\hline Turista: T & Seguridad: S & $\begin{array}{l}\text { Asociaciones de } \\
\text { turismo: AS }\end{array}$ & Política y planeación: P2 \\
\hline Tipología de turismo: T2 & Decisión de destino: D2 & Agencia de turismo: AT & Participación de actores: PA \\
\hline Medios de promoción: MP & & Proveedores: $\mathrm{P}$ & Gestión del destino: GD \\
\hline \multirow[t]{7}{*}{ Oferta: O } & & Localización: L & Interrelación cultural: IC \\
\hline & & Accesibilidad: A & Atractivo de turismo: AT \\
\hline & & Infraestructura: I & Empleabilidad: E \\
\hline & & \multicolumn{2}{|l|}{ Entorno rural: ER } \\
\hline & & \multicolumn{2}{|l|}{$\begin{array}{l}\text { Profesionales en } \\
\text { turismo:PT }\end{array}$} \\
\hline & & \multicolumn{2}{|l|}{ Rutas: R } \\
\hline & \multicolumn{3}{|c|}{ Experiencia única: EU } \\
\hline
\end{tabular}


Modelo de cadena de valor en turismo rural comunitario.

$$
f(E U)=\frac{V I A T+V I A I}{V D A T+V D A I}
$$

Función 1. Experiencia única.

$$
f(V I A T)=\int T+T^{2}+M P+O
$$

La función experiencia única responde a la sumatoria de variables independientes y dependientes con enfoque de aspectos tangibles e intangible.

Función 2. Los factores que inciden en la formula son variables independientes con aspectos tangibles.

$$
f(V I A I)=\int S+D^{2}
$$

Función 3. Los factores que definen la formula son variables independientes con aspectos intangibles.

$$
f(V D A T)=\int A S+A T+P+L+A+I+E R+P T+R
$$

Función 4. La función está determinada por variables dependientes de aspectos tangibles

$$
f(V D A I)=\int P^{2}+P A+G D+I C+A T+E
$$

Función 5. La función está representada por variables dependientes de aspectos intangibles Dentro del análisis de variables independientes y dependientes con enfoque de aspectos tangibles e intangibles. Se construyó el modelo de cadena de valor en turismo rural comunitario a partir de aspectos que se consideran deben articular para desarrollo óptimo de la cadena de valor.

\section{Resultados}

En la tabla 4., se representa la tipología de turismo ofertado y en la tabla 5, se encuentran relacionados el indicador de accesibilidad en el turismo:

Tabla 4

¿Qué tipo de turismo ofrece?

\begin{tabular}{llr|r|r|r} 
& & & \multicolumn{2}{c}{$\begin{array}{c}\text { Porcentaje } \\
\text { válido }\end{array}$} & $\begin{array}{c}\text { Porcentaje } \\
\text { acumulado }\end{array}$ \\
\hline Válido & Turismo de sol y playa & 5 & 23,8 & 25,0 & 25,0 \\
\cline { 2 - 6 } & Turismo natural & 12 & 57,1 & 60,0 & 85,0 \\
\cline { 2 - 6 } & Turismo de salud & 3 & 14,3 & 15,0 & 100,0 \\
\cline { 2 - 6 } & Total & 20 & 95,2 & 100,0 & \\
\hline Perdidos & Sistema & 1 & 4,8 & & \\
\hline Total & & 21 & 100,0 & & \\
\hline
\end{tabular}


Figura 1

Tipología de turismo

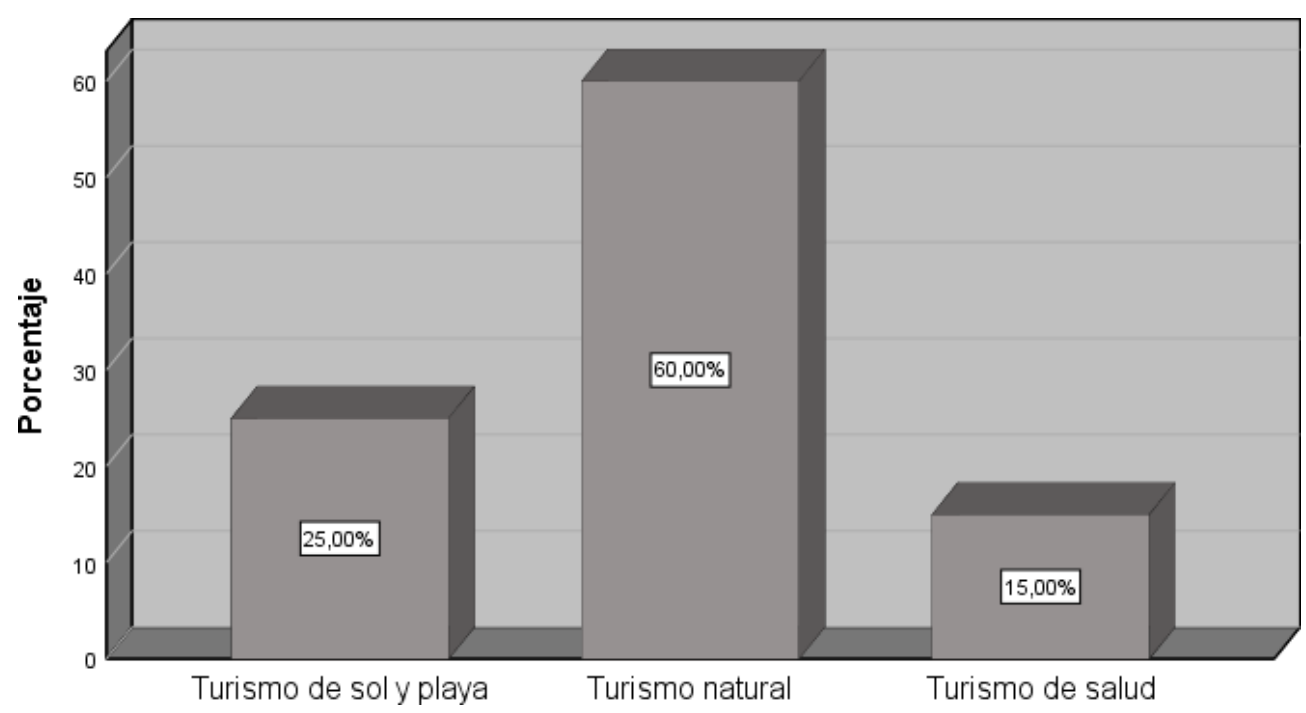

Tabla 5

¿Dentro de los servicios que ofrece se encuentran actividades de turismo accesible?

\begin{tabular}{llr|r|r|r} 
& Frecuencia & Porcentaje & $\begin{array}{c}\text { Porcentaje } \\
\text { válido }\end{array}$ & $\begin{array}{c}\text { Porcentaje } \\
\text { acumulado }\end{array}$ \\
\hline Válido & Si & 6 & 28,6 & 30,0 & 30,0 \\
\cline { 2 - 6 } & No & 14 & 66,7 & 70,0 & 100,0 \\
\cline { 2 - 7 } & Total & 20 & 95,2 & 100,0 & \\
\hline Perdidos & Sistema & 1 & 4,8 & & \\
\hline Total & 21 & 100,0 & & \\
\hline
\end{tabular}


Figura 2

Accesibilidad de turismo

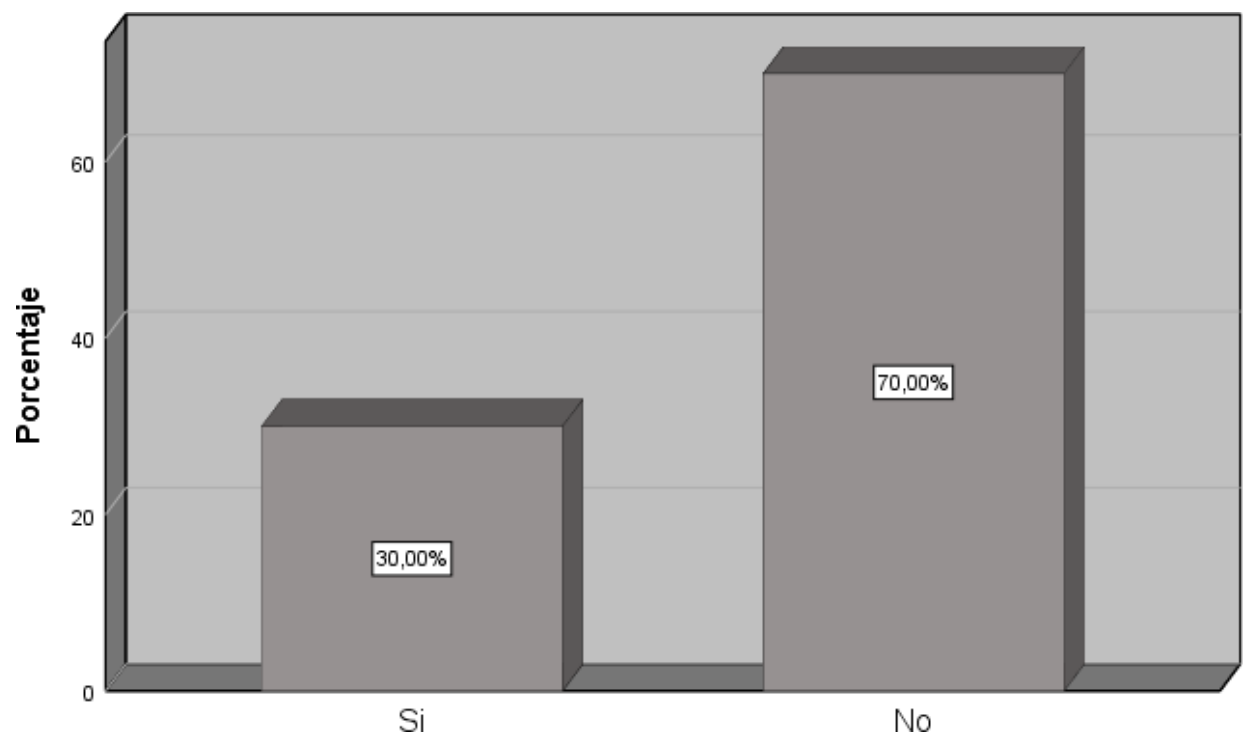

Colombia se ubica en la franja intertropical por la cordillera de los Andes, atravesada de sur a norte destacándose por la mega diversidad de la región, conformada por las cordilleras oriental, occidental y central, al mismo tiempo, ofrece variedad de ecosistemas naturales endémicos para el disfrute sostenible de la humanidad. En el análisis de resultados, el turismo fue clasificado en tipología y oferta de turismo; Es decir que, el turismo natural representa el $50 \%$ de la demanda total. El siguiente atractivo fue el turismo de sol y playa con el $29.2 \%$ de clasificación, el turismo cultural con el $16.7 \%$ y por último, el turismo de salud con el $4.2 \%$.

De acuerdo con esto, se obtuvo información a través de guías especializados y agencias de turismo para conocer el estado actual de turismo rural comunitario. Se estimó que la oferta de turismo a nivel nacional se encuentra en un $75 \%$ y el $25 \%$ en turismo internacional. Se puede confirmar que, gracias a la diversidad de fauna y flora, el país es atractivo turístico a nivel mundial (Andrade-C, 2011). La localización es factor de influencia en el desarrollo de turismo, entre las zonas de potencial turístico rural comunitario está la capital del país (Bogotá) representada con el 66.7\%, seguido del departamento de Boyacá con el $45.8 \%$, en la Guajira y Amazonia con el 37.5\%, Antioquia y Neiva 33.3 entre otros encontrados debajo del 30\%. De manera simultánea, las agencias y guías de turismo consultados afirmaron conocer acerca de turismo rural comunitario en un $75 \%$ y el $25 \%$ expresa desconocimiento de éste tipo de turismo.

En este orden de ideas, existen factores de significancia que tendría en cuenta para ofertar turismo rural comunitario, se destacan aspectos importantes como la calidad del servicio con el $79 \%$, interrelación intercultural con $66 \%$, entorno rural $62 \%$, ruta $54 \%$, localización $50 \%$, personal profesional 50\%, infraestructura $45 \%$.

De lo anterior, se aborda la accesibilidad del turismo como la capacidad de que todos puedan acceder y hacer uso de los atractivos turísticos sin ninguna limitante, se debe abordar la accesibilidad del turismo, definido como la capacidad de que todos puedan acceder y hacer uso de atractivos turísticos; independientemente de capacidades físicas, cognitivas, y de madurez. Sólo el $25 \%$ de los servicios que ofrecen cumplen con indicaciones de accesibilidad y el $75 \%$ no garantiza accesibilidad en el desarrollo de los mismos. Estos resultados muestran a que lo rural resignifica, espacios rurales como espacios territoriales que van más allá de lo agropecuario y en donde se privilegia la pluriactividad de los sistemas de producción 
y la multifuncionalidad de los espacios territoriales. Lo que se ha denominado como "enfoque territorial del desarrollo rural" (DRET), que Schejtman y Berdegué (2004) resumen en el concepto de desarrollo territorial rural (DTR) y lo definen como "un proceso de transformación productiva e institucional en un espacio rural determinado, cuyo fin es reducir la pobreza rural". (Pérez, 2010). Finalmente, el turismo juega un papel importante dentro de la comunidad y la falta de información, obstaculizando el conocimiento acerca de beneficios para llevar a cabo el desarrollo del turismo, en algunos casos la comunidad no tiene interés en hacer parte de estos proyectos (Gebreegziabher, 2018).

\section{Conclusiones}

El turismo rural comunitario es una estrategia sustentable como medida de adaptación y mitigación para hacer frente al cambio climático, favoreciendo al ambiente a través del almacenamiento de carbono, convirtiendo a los ecosistemas en reservorios que benefician a las personas. En consonancia con los resultados encontrados es necesario ahondar en la innovación del desarrollo rural comunitario, que conduzca a la identificación de productos y servicios con valores diferenciados según sus territorios que evidencia un $62 \%$ rural pero acompañados de una interculturalidad del $66 \%$, valor que diversifica y aumenta el turismo rural comunitario al fusionarlo al turismo gastronómico, al turismo de sol y playa y al turismo natural.

En consecuencia, la política pública administra estrategias garantes de bienestar social por medio de la administración de recursos que suplan factores socioeconómicos de la población. Está categoría marcada en la tipología de turismo natural, salvaguarda el patrimonio ambiental, social y cultural, creando valor agregado a través de intercambio en experiencias únicas que mejoren la percepción social. Es necesario, articular las variables que inciden en la creación de valor en torno a la gestión de proyectos que aseguren ingresos y disminuyan la incertidumbre de las dinámicas agropecuarias. Es de vital importancia, la inclusión de poblaciones étnicas y campesinas en la distribución equitativa, derivando procesos de empoderamiento en la preservación y conservación de recursos naturales en países que albergan el mayor índice de biodiversidad.

La creación de valor en la cadena del turismo rural comunitario debe basar su desarrollo en las siguientes líneas de trabajo, las cuales deben ser abordas desde el dialogo de saberes y la investigación acción participación que permite el rescate de los valores que se encuentran en las comunidades nativas y de amplia diversidad en dialectos y costumbres que resaltarían el desarrollo territorial rural, incluso dando mayores valor a este conjunto de atractivos en el turismo de sol y playa, puede enriquecerse y conducir a estancias mas duraderas y por ende mayor generación de ingresos locales y estos aspectos comunitarios que se desarrollan en las áreas rurales se involucran en el destino turístico y claramente requiere de la gestión apropiada para la identificación de productos locales (gastronomía, culturas, dialectos, lenguas y biodiversidad).

De igual manera el turismo rural comunitario se evidencia de acuerdo a los segmentos consultados como el turismo de sol y playa, que goza de mayores infraestructuras que el turismo de natural, este aspecto es fundamental seguir investigando toda vez que se requiere, desarrollo en innovación en la infraestructura que conduzca a: materiales sostenibles, sitios de menor afectación, incorporación de tecnologías apropiadas en el manejo de residuos y acceso a la energía renovable; y de igual manera la búsqueda del desarrollo de pequeñas empresas y medianas empresa de turismo rural, con un marco regulatorio, con procesos de formación y capacitación en tal medida que se logre el empoderamiento para lograr niveles de gestión óptimos, buscando el desarrollo territorial rural de forma integral. La sostenibilidad del destino es fundamental no dejarla de lado en el análisis toda vez que si no se incorporar como variable de gestión, no tendría la sustentabilidad de la actividad económica y sede sus comunidades asentadas. 


\section{Referencias bibliográficas}

Altinay, L., Sigala, M., \& Waligo, V. (2016). Social value creation through tourism enterprise. Tourism Management, 404 - 417. DOI: https://doi.org/10.1016/j.tourman.2015.12.011.

Andrade-C, M. (2011). estado del conocimiento de la biodiversidad en colombia y sus amenazas. consideraciones para fortalecer la interacción ciencia-política. Medio ambiente, 492 - 504.

Ashish , K., Umesh , C., \& P. Vigneswara, I. (2017). Social Media Usage for Tourism: A Case of Rajasthan Tourism. Procedia Computer Science, Volume 122, Pages 751-758. DOI: https://doi.org/10.1016/j.procs.2017.11.433.

Becken, S., Zammit, C., \& Hendrikx, J. (2014). Developing climate change maps for tourism essential information or awareness raising. Journal of Travel Research, DOI: 10.1177/0047287514528286.

Blasco López, M., Recuero Virto, N., Aldas Manzano, J., \& García-Madariaga Miranda, J. (2018 ). Residents' attitude as determinant of tourism sustainability: The case of Trujillo. Journal of Hospitality and Tourism Management, (35) 36 - 45 DOI: https://doi.org/10.1016/j.jhtm.2018.02.002.

Butler , J., \& S. Dyer , J. (2014a). Introduction to Multiattribute Utility Theory. Policy, (46), 1-13.DOI: https://doi.org/10.1002/9780470400531.eorms1091.

Butler, J., Young, J., McMyn, I., Leyshon, B., Grahamel, I., Walkerf, I., . . Warburtoni, C. (2015b). Evaluating adaptive co-management as conservation conflict resolution: Learning from seals and salmon. Journal of Environmental Management, (160) Pages 212-225. DOI :https://doi.org/10.1016/j.jenvman.2015.06.019.

Bynum Boleya, B., \& Gard McGeheeb, N. (2014). Measuring empowerment: Developing and validating the Resident Empowerment through Tourism Scale (RETS). Tourism Management, Volume 45, December 2014, Pages 85-94. DOI: https://doi.org/10.1016/j.tourman.2014.04.003.

Chaperon, S., \& Bramwell, B. (2013). Dependency and agency in peripheral tourism development. Annals of Tourism Research, 40, 132-154.

Carneiro, M. J., Lima, J., \& Silva, A. L. (2015). Landscape and the rural tourism experience: identifying key elements, addressing potential, and implications for the future. Journal of Sustainable Tourism, 23(8-9), 1217-1235.

Clemente Soler , J., Bote Díaz , M., \& Sánchez Vera, P. (2018). El turismo social accesible como nuevo modelo turístico. Cuadernos de Turismo,, № 41, pp. 139-159. DOI: http://dx.doi.org/10.6018/turismo.41.326981

Corigliano, M. A., Viganò, G., \& Mottironi, C. (Eds.). (2015). Tourism in the Alps: governing sustainability. Ministero dell'Ambiente

Conway , D., \& Mustelin, J. (2014). Strategies for improving adaptation practice in developing countries. Nature Climate Change , (4) 339 - 342.

Cuenca, M., \& Prat, A. (2012). Ocio experiencial: antecedentes y características. Arbor. Ciencia, Pensamiento y Cultura, vol. 188, no 756, pp. 265-281.

Dolnicar, S., Yanamandram, V., \& Cliff, K. (2012). The contribution of vacations to quality of life. Annals of Tourism Research, 59 - 83. DOI :https://doi.org/10.1016/j.annals.2011.04.015.

Enquist, B., Petros Sebhatu, S., \& Johnson,, M. (2015). Transcendence for business logics in value networks for sustainable service business. Journal of Service Theory and Practice,, 181-197. DOI: https://doi.org/10.1108/JSTP-09-2013-0189.

Flórez Ñustes , J., \& Laguna Barragán, A. (2017). El circo social: una propuesta de tejido comunitario. Inlcusión \& Desarrollo, 56. DOI: http://dx.doi.org/10.26620/uniminuto.inclusion.4.1.2017 
Fotis, J., Buhails, D., \& Rossides, N. (2012). Social Media Use and Impact during the Holiday Travel Planning Process. Information and Communication Technologies in Tourism , 1 - 2 .

Fiorello, A., \& Bo, D. (2012). Community-based ecotourism to meet the new tourist's expectations: an exploratory study. Journal of Hospitality Marketing and Management, 21(7), 758-778

Gravari-Barbas, M. (2018). Tourism as a heritage producing machine. Tourism Management Perspectives, Volume 26, April 2018, Pages 5-8.

Garcés, J., \& Ferri , M. (2012). Establecimiento de los criterios de accesibilidad universal a los recursos turísticos. Proyecto: Investigación para la mejora del conocimiento en turismo social accesible para todos en España (SOCIALTOURISM).

García Henche, B., \& Salvaj Carrera, E. (2017). Asociacionismo, redes y marketing en la transformación hacia el turismo experiencial. el caso del barrio de las letras. madrid. Cuadernos de Turismo, № 40, (2017); pp. 315-338. DOI: http://dx.doi.org/10.6018/turismo.40.309731.

Gebreegziabher Woldu, M. (2018). Community based tourism in Lake Tana growth corridor of the Amhara region of Ethiopia: The missing link among stakeholders and implicationsto tourism industry. Woldu, Cogent Social Sciences, 9 - 14. DOI: DOI: 10.2139/ssrn.2880075.

Gómez Martín , M., Armesto López , X., \& Cors Iglesias , M. (2017). Percepción del cambio climático y respuestas locales de adaptación: el caso del turismo rural. Cuadernos de Turismo, № 39, pp. 287-310. DOI: http://dx.doi.org/10.6018/turismo.39.290571.

Goodwin, H., \& Santilli, R. (2009). Community-based tourism: A success? ICRT Occasional Paper 11. GTZ. ICRT Occasional Paper 11. GTZ.

Jaafar, M., \& Md Noorb, S. (2015). Perception of young local residents toward sustainable conservation programmes: A case study of the Lenggong World Cultural Heritage Site. Tourism Management, Pages 154-163. DOI: https://doi.org/10.1016/j.tourman.2014.10.018.

Kaplan, M. (2010). Users of the world, unite! The challenges and opportunities of Social Media. Business Horizons, Volume 53, Issue 1, Pages 59-68. DOI: https://doi.org/10.1016/j.bushor.2009.09.003.

Kline, C., Shah, N., \& Rubright, H. (2014). Applying the Positive Theory of Social Entrepreneurship to Understand Food Entrepreneurs and Their Operations. Journal Tourism Planning \& Development, 330 - 342. DOI: https://doi.org/10.1080/21568316.2014.890126.

Klint, L., Jiang, M., Law, A., Delacy, T., Filep, S., Calgaro, E., Harrison, D. (2012). Dive tourism in Luganville, Vanuatu: shocks, stressors, and vulnerability to climate change. Tourism in Marine Environments, № 8(1), pp. 91-109.

Kyungmi , K., \& Muzaffer , U. (2013). How does tourism in a community impact the quality of life of community residents? Tourism Management, (36) 527 - 540. DOI: http://dx.doi.org/10.1016/j.tourman.2012.09.005.

Lane, B., \& Kastenholz, E. (2015). Rural tourism: the evolution of practice and research approaches - towards a new generation concept? Journal of Sustainable Tourism, (23) 8 -9.

Liang, Z.-X., \& Hui, T.-K. (2016). Residents' quality of life and attitudes toward tourism development in China. Tourism Management, 56 - 67. DOI : https://doi.org/10.1016/j.tourman.2016.05.001.

Lin, Z., Chen, Y., \& Filieric, R. (2017). Resident-tourist value co-creation: The role of residents' perceived tourism impacts and life satisfaction. Tourism Management, 436 - 442 . DOI: https://doi.org/10.1016/j.tourman.2017.02.013.

Liua, J., Qu, H., Huang, D., Chen, G., Yue, X., Zhao, X., \& Lianga, Z. (2014). he role of social capital in encouraging residents' pro-environmental behaviors in community-based ecotourism. Tourism Management, (48)190201.DOI: https://doi.org/10.1016/j.tourman.2013.08.016. 
López-González, J. (2018). Exploring discourse ethics for tourism transformation. TOURISM - An International Interdisciplinary Journal, 66(33), 269-281.

Minciu, R. (2004). Economia Turismului. Edi ia a III-a, Editura Uranus, Bucure ti. Economia Turismului.

Minnaert, L. (2014). Social tourism participation: The role of tourism inexperience and uncertainty. Tourism Management, (40) 282 - 289.DOI: https://doi.org/10.1016/j.tourman.2013.07.002.

Mustafa, M., \& Tayeh, S. (2011). The impacts of tourism development on the archaeological site of Petra and local communities in surrounding villages. Asian Social Science, , 7(8), 88 - 96.

Nunkoo, R., \& So, K. (2015). Residents' support for tourism testing alternative structural models. Journal of Travel Research, (5) $1-15$.

Ocampo González, A. (2017). Producción social del espacio: el capital y las luchas sociales en la disputa territorial. Inclusión y desarrollo, 87. DOI: http://dx.doi.org/10.26620/uniminuto.inclusion.1.1.2014.

Onyango, E., \& Kesa, E. (2018). The fourth industrial revolution: Implications for hotels in South Africa and Kenya. TOURISM - An International Interdisciplinary Journal, 349-353.

Organización Mundial del Turismo y Organización de los Estados Americanos. (2018). Él turismo y los Objetivos de Desarrollo Sostenible - Buenas prácticas en las Américas, OMT, Madrid.

Organización Mundial del Turismo (2019), Definiciones de turismo de la OMT, OMT, Madrid.

Palutikof, J., Parry, M., Stafford Smith, M., Boulter, S., \& Waschka, M. (2013). The past, present and future of adaptation: setting the context and naming the challenges. In: Palutikof, J., Boulter, S.L., Ash, A.J., Stafford Smith, M., Parry, M., Waschka, M., Guitart, D. (Eds.), Climate Adaptation Futures., 1 - 29.

Perez, Samuel, El valor estratégico del turismo rural como alternativa sostenible de desarrollo territorial rural, (2010). Revista Agronomía Colombiana 28(3), 507-513.

Prabhakarana, S., Naira, V., \& Ramachandranb, S. (2014). 5th Asia Euro Conference 2014 - Community participation in rural tourism: Towards a conceptual. Procedia - Social and Behavioral Sciences, (144 ) 290 $-295$.

Po-Hsin , L., Morrison-Saunders, A., \& Grimstad, S. (2017). Operating small tourism firms in rural destinations: A social representations approach to examining how small tourism firms cope with non-tourism induced changes. Tourism Management , (58) 164 -174.

Pulido-Fernandez, J., Andrades-Caldito, L., \& Sanchez- Rivero, M. (2015). Is sustainable tourism an obstacle to the economic performance of the tourism industry? Evidence from an international empirical study. Journal of Sustainable Tourism, 23(1), 47- 64. DOI: https://doi.org/10.1080/09669582.2014.909447.

Rasoolimanesha, S., Jaafara, M., Ned , K., \& Ramayahc, T. (2015). A revised framework of social exchange theory to investigate the factors influencing residents' perceptions. Tourism Management Perspectives, Volume 16, Pages 335-345.DOI: https://doi.org/10.1016/j.tmp.2015.10.001.

Rihova, I., Buhalis , D., Moital , M., \& Gouthro , M.-B. (2015). Conceptualising Customer-to-customer Value Cocreation in Tourism. International Journal Tourism Research, 356 -363. DOI: https://doi.org/10.1002/jtr.1993.

Ritchie , J., \& Hudson, S. (2009). Understanding and meeting the challenges of consumer/tourist experience research. International Journal Tourism Research, 111-126. DOI: https://doi.org/10.1002/jtr.721 .

Rodima-Taylor, D., F.Olwig, M., \& Chhetrid, N. (2012). Adaptation as innovation, innovation as adaptation: An institutional approach to climate change. Applied Geography, (33) Pages 107-111. DOI : https://doi.org/10.1016/j.apgeog.2011.10.011. 
Rodriguez-Giron, S., \& Vanneste, D. (2018). Determinantes para el acceso a los mercados internacionales de destinos turísticos: el caso del sur de Ecuador y Alemania. TOURISM - An International Interdisciplinary Journal, 66(3), 282-301.

Salvatore, R. (2015). Between nature and landscape: The role of community towards an active conservation in protected areas. In R. Gambino, \& A. Peano (Eds.). Nature policies and landscape policies , (pp. 223-231).

Salvatore, R., Chiodo, E., \& Fantini, A. (2018). Tourism transition in peripheral rural areas: Theories, issues and strategies. Annals of Tourism Research, Volume 68, Pages 41-5. DOI: https://doi.org/10.1016/j.annals.2017.11.003.

Scaglione, M., Marx, S., \& Johnson, C. (2011). Tourism and poverty alleviation approaches: A case study comparison. Global imbalances-Poverty alleviation, (5, pp. 207-226).

Sedgley, D., Pritchard, A., \& Morgan, N. (2012). 'Tourism poverty' in affluent societies: voices from inner-city London. Tourism Management, 33, 951 - 960

Sharpley, R. (2014). Host perceptions of tourism: A review of the research. Tourism Management, 37 - 49. DOI: https://doi.org/10.1016/j.tourman.2013.10.007.

Sørensen, F., \& Jensen, J. (2015). Value creation and knowledge development in tourism experience encounters. Tourism Management , (46) 336 - 346.

Sørensen, F., Mattsson, J., \& Sundbo, J. (2013). Organisational conditions for service encounter based innovation. Research Policy, (42), $1446-1456$.

Stylidis, D., Biran, A., Sit, J., \& Szivas, E. (2014). Residents' support for tourism development: The role of residents' place image and perceived tourism imimpacts. Tourism Management, Volume 45, Pages 260-274 Pages 260-274 DOI :https://doi.org/10.1016/j.tourman.2014.05.006

UNWTO \& SNV. (2010). Manual on tourism and poverty alleviation, practical steps for destinations. Madrid: World Tourism.Organización Mundial del Turismo y Organización de los Estados Americanos (2018)

Ursache, M. (2015). Tourism - significant driver shaping a destinations heritage. Procedia - Social and Behavioral Sciences 188 ( 2015 ) 130 - 137, 188130 - 137. DOI :https://doi.org/10.1016/j.sbspro.2015.03.348.

Vargo, S., \& Lusch , R. (2011). It's all B2B...and beyond: Toward a systems perspective of the market. Industrial Marketing Management, Pages 181-187. DOI: https://doi.org/10.1016/j.indmarman.2010.06.026.

Wang, S., \& Chen, J. (2015). The influence of place identity on perceived tourism impacts. Annals of Tourism Research, 52, 16 - 28. DOI : https://doi.org/10.1016/j.annals.2015.02.016.

Williams, A., \& Baláz, V. (2012). Migration, risk and uncertainty: theoretical perperspectives. Population, Space and Place,, 18, 167- 180.

Woo, E., Kim, H., \& Uysal, M. (2015 ). Life satisfaction and support for tourism development. Annals of Tourism Research, 50, 84 - 9. DOI: https://doi.org/10.1016/j.annals.2014.11.001.

Zahra, S., Gedajlovic, E., Neubaumc, D., \& Shulmand, J. (2009). A typology of social entrepreneurs: Motives, search processes and ethical challenges. Journal of Business Venturing, 519 - 532. DOI: https://doi.org/10.1016/j.jbusvent.2008.04.007. 